\title{
Fighting the Curse of Dimensionality in First- Principles Semiclassical Calculations: Non-Local Reference States for Large Number of Dimensions
}

\section{Citation}

Ceotto, Michele, Gian Franco Tantardini, and Alán Aspuru-Guzik. 2011. Fighting the curse of dimensionality in first-principles semiclassical calculations: Non-local reference states for large number of dimensions. Journal of Chemical Physics 135(21): 214108.

\section{Published Version}

doi:10.1063/1.3664731

\section{Permanent link}

http://nrs.harvard.edu/urn-3:HUL.InstRepos:8438168

\section{Terms of Use}

This article was downloaded from Harvard University's DASH repository, and is made available under the terms and conditions applicable to Open Access Policy Articles, as set forth at http:// nrs.harvard.edu/urn-3:HUL.InstRepos:dash.current.terms-of-use\#OAP

\section{Share Your Story}

The Harvard community has made this article openly available.

Please share how this access benefits you. Submit a story.

\section{Accessibility}




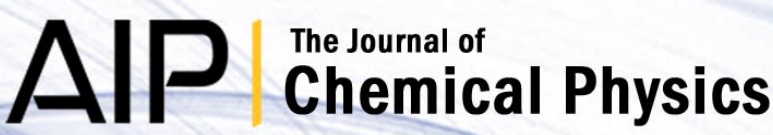

Fighting the curse of dimensionality in first-principles semiclassical calculations: Non-local reference states for large number of dimensions

Michele Ceotto, Gian Franco Tantardini, and Alán Aspuru-Guzik

Citation: J. Chem. Phys. 135, 214108 (2011); doi: 10.1063/1.3664731

View online: http://dx.doi.org/10.1063/1.3664731

View Table of Contents: http://jcp.aip.org/resource/1/JCPSA6/v135/i21

Published by the American Institute of Physics.

Additional information on J. Chem. Phys.

Journal Homepage: http://jcp.aip.org/

Journal Information: http://jcp.aip.org/about/about_the_journal

Top downloads: http://jcp.aip.org/features/most_downloaded

Information for Authors: http://jcp.aip.org/authors

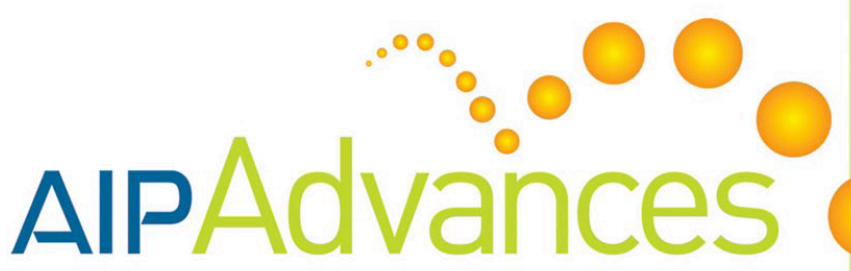

Explore AIP's new open-access journal

Article-level metrics now available

Join the conversation! Rate $\&$ comment on articles 


\title{
Fighting the curse of dimensionality in first-principles semiclassical calculations: Non-local reference states for large number of dimensions
}

\author{
Michele Ceotto, ${ }^{1, a)}$ Gian Franco Tantardini, ${ }^{1,2}$ and Alán Aspuru-Guzik ${ }^{3}$ \\ ${ }^{1}$ Dipartimento di Chimica Fisica ed Elettrochimica, Università degli Studi di Milano, via Golgi 19, \\ 20133 Milano, Italy \\ ${ }^{2}$ Istituto CNR di Scienze e Tecnologie Molecolari, via Golgi 19, 20133 Milano, Italy \\ ${ }^{3}$ Department of Chemistry and Chemical Biology, Harvard University, 12 Oxford Street, Cambridge, \\ Massachusetts 02138, USA
}

(Received 20 September 2011; accepted 9 November 2011; published online 6 December 2011)

\begin{abstract}
Semiclassical methods face numerical challenges as the dimensionality of the system increases. In the general context of the theory of differential equations, this is known as the "curse of dimensionality." In the present manuscript, we apply the recently-introduced multi-coherent states semiclassical initial value representation (MC-SC-IVR) approach to extend the applicability of first-principles semiclassical calculations. The proposed strategy involves the use of non-local coherent states with the goal of increasing accuracy in the Fourier transforms, and on the other hand, allows for the selection of peaks of different frequencies. The ability to filter desired peaks is important for analyzing the power spectra of complex systems. The MC-SC-IVR approach allows us to solve a 19-dimensional test system and to resolve on-the-fly the power spectra of the formaldehyde molecule with very few classical trajectories. () 2011 American Institute of Physics. [doi:10.1063/1.3664731]
\end{abstract}

\section{INTRODUCTION}

The simulation of the quantum dynamics of molecular vibrations is still mostly limited to systems with few degrees of freedom. In a few cases, by means of clever approximations, the dynamics of systems of a large number of degrees of freedom have been simulated. ${ }^{1,2}$ The limitation to relatively low dimensionality is given not only by issues related to the challenges of propagating the quantum dynamics in many-dimensions, but also by the construction of an electronic potential energy surface (PES). For many-dimensional systems, due to computational limitations, the PES fitting procedure results in a sometimes drastic trade-off of computational efficiency vs accuracy of the fit. Several techniques have been developed ${ }^{3}$ to ease these difficulties. Nowadays, first principles molecular dynamics (FPMD), or ab initio molecular dynamics (AIMD) is becoming a more attractive and useful technique to avoid computing PESs explicitly. During a FPMD simulation, the dynamics is calculated at each time step on-the-fly, i.e., directly from an electronic structure calculation. Examples of these approaches include the Born-Oppenheimer molecular dynamics methods (BOMD), ${ }^{4-8}$ the extended Lagrangian molecular dynamics approaches (ELMD), ${ }^{9-15}$ and the $a b$ initio multiple spawning (AIMS) algorithm. ${ }^{16}$

The fundamental limitation for simulating quantum dynamics on a classical computer is due to the "curse of dimensionality". To make progress, a finite set of basis function is usually employed to represent a wave function. A suitable combination of these functions are used to represent at each time step the quantum wavefunction. However, formally, the dimensionality of this set scales exponentially.

\footnotetext{
a)Electronic mail: michele.ceotto@unimi.it.
}

Quantum dynamical approaches that avoid this unfavorable scaling have been developed, ${ }^{17}$ however these are still not coupled with on-the-fly first principles approaches. Therefore, current MCTDH simulations are restricted to the case of fitted PES. It might seem like a futuristic alternative, but quantum dynamics on quantum computers do not suffer from this exponential curse, ${ }^{18}$ and have recently been successfully simulated experimentally using an NMR approach. ${ }^{19}$

In order to approximately bypass the dimensionality restrictions mentioned above, semiclassical molecular dynamics has been an attractive alternative. ${ }^{20-23}$ Regarding the first limitation about the requirement of a fitted PES, semiclassical molecular dynamics has the advantage of relying on classical trajectories obtained from first-principles simulations of nuclear dynamics. In order to bypass the second limitation associated with the dimensionality of the basis set, the semiclassical initial value representation (SC-IVR) (Ref. 24) using the Heller-Hermann-Kluk-Kay (HHKK) propagator has been developed over the last 30 years. ${ }^{25-28}$ In HHKK SCIVR, the basis set is given by a combination of coherent states and the propagator is estimated via Monte Carlo integration in phase space. In this way, the scaling with the dimensionality is greatly reduced. ${ }^{29}$

The main thrust of research in SC-IVR theories is that of being able to simulate more and more complex molecular systems. On-the-fly dynamical SCI-VR simulations are still restricted to situations of few degrees of freedom. An obvious possible route to take is to employ computational power by brute force, using more and more trajectories as the dimensionality of the system grows. This is not scalable in general and computational challenging for a first principles approach, where forces and other dynamical components are calculated directly from electronic structure packages. In this paper, we will present a method that allows SC-IVR to go to a larger 
number of dimensions without requiring additional trajectories. Even this method eventually run out of steam.

For this work, the main application of the proposed firstprinciples semiclassical initial value representation (FP-SCIVR) molecular dynamics will be the calculation of vibrational spectral density obtained after propagating an initial trial or reference state $|\chi\rangle,{ }^{30}$

$$
I(E) \equiv\langle\chi|\delta(\hat{H}-E)| \chi\rangle=\sum_{n}\left|\left\langle\chi \mid \psi_{n}\right\rangle\right|^{2} \delta\left(E-E_{n}\right) .
$$

Here, $\left\{\left|\psi_{n}\right\rangle\right\}$ and $\left\{E_{n}\right\}$ are the collections of exact eigenstates and eigenvalues of the Hamiltonian $\hat{H}$. The spectrum of Eq. (1) contains a wealth of quantum mechanical information about the simulated system: The peaks are located at the positions of the eigenvalues and their intensity is proportional to the square of the overlap between the trial state $|\chi\rangle$ with the eigenvectors of the PES. Replacing the Dirac delta function of Eq. (1) by its Fourier representation, one can more conveniently achieve the same spectrum with the following time dependent representation, ${ }^{31}$

$$
I(E)=\frac{1}{2 \pi \hbar} \int_{-\infty}^{+\infty}\left\langle\chi\left|e^{-i \hat{H} t / \hbar}\right| \chi\right\rangle e^{i E t / \hbar} d t
$$

where the spectrum is written in terms of the Fourier transform of the survival probability. As mentioned above, in the SC-IVR formulation of the quantum propagator the bracket in Eq. (2) can be cast as a Monte Carlo phase space integration. In this context, the brute force approach that we refereed to earlier, consists of sampling an increasing number of trajectories as a function of the number of dimensions to achieve an efficient Monte Carlo estimate. An alternative approach to gather statistics for the phase space integration is that of taking longer time trajectories. This is computationally much more convenient if one inserts a time averaging filter before the phase space integration. ${ }^{32-34} \mathrm{We}$ find this formulation of the SC-IVR extremely useful, since the number of phase space trajectories can be reduced to a set of very few. At the same time, high resolution spectra (up to $1 \mathrm{~cm}^{-1}$ ) can be achieved using such trajectories. So far, these simulations have been restricted to very few dimensions. ${ }^{21-23}$

In order to extend the applicability of the method, we explore the development of improved reference states. The reference state is usually defined as the direct product of the harmonic ground state approximation of each degree of freedom, or more generally as a direct product of one-dimensional wavefunctions,

$$
|\chi\rangle=\left|\psi\left(x_{1}\right)\right\rangle\left|\psi\left(x_{2}\right)\right\rangle \ldots\left|\psi\left(x_{F}\right)\right\rangle .
$$

To obtain a sharp and complete spectral density from Eq. (2) a recurring overlap of the wavefunction with the reference function is essential in order to have an non-zero integrand to be Fourier transformed, and obtain a signal. In other words, if the trajectory does not return to regions of phase space with large overlap with the initial wave packet, the spectral density obtained is either broadened or very noisy. As mentioned above possible way to move forward is to use a more sophisticated reference state that has larger overlap with the recurring trajectory. As an informative example of a system with many degrees of freedom one can think of a system with a separable Hamiltonian. In this case, the integrand of Eq. (2) is the direct product of the overlap in each dimension,

$$
\left\langle\psi\left(x_{1}\right)\left|e^{-i \hat{H}_{1} t / \hbar}\right| \psi\left(x_{1}\right)\right\rangle \ldots\left\langle\psi\left(x_{F}\right)\left|e^{-i \hat{H}_{F} t / \hbar}\right| \psi\left(x_{F}\right)\right\rangle .
$$

The direct product in Eq. (4) is only significant for the Fourier transform, when the multidimensional classical trajectory visits a number of time points $\left(x_{1}(t), x_{2}(t), \ldots, x_{F}(t)\right)$ that are sufficiently near to the starting one $\left(x_{1}(0), x_{2}(0), \ldots, x_{F}(0)\right)$. Since in the semiclassical formulation, these are phase space points and the basis set is given by the direct product of one dimensional coherent states, i.e., with an exponentiallydecaying density, then the contributions to the Fourier integral arise when the recurrence happens to be within the Gaussian spreading of the coherent state. This short range overlap can be partially tamed by using smaller Gaussian coefficients for the propagating state, i.e., wider spreading.

Thus from Eq. (4), it is clear how the main issue is that the coherent states should be significantly overlapped almost simultaneously for all dimensions. This is a rare event when a few-trajectory simulation is performed and the dimensionality increases.

In Sec. II, we briefly review the time averaging SC-IVR formulation for power spectra calculations. In Sec. III, implementations of our recently developed Multiple Coherent States SC-IVR method are presented for dealing better with many dimensions and in Sec. IV these are applied to analytical models. Finally, in Sec. V the method is applied to the first-principles simulation of the formaldehyde molecule. Conclusions are drawn in Sec. VI.

\section{REVIEW OF THE TIME-AVERAGING SEMICLASSICAL INITIAL VALUE REPRESENTATIONS}

For the time-dependent calculation of the power spectra in Eq. (2) one can approximate the propagator semiclassically. The initial value representation formulation (SCIVR) converts the semiclassical integration over all classical trajectories at given end positions into a more convenient $F$-dimensional phase space integration as follows:

$$
\begin{aligned}
e^{-i \hat{H} t / \hbar}= & \frac{1}{(2 \pi \hbar)^{F}} \int d \mathbf{p}(0) \int d \mathbf{q}(0) C_{t}(\mathbf{p}(0), \mathbf{q}(0)) \\
& \times e^{i S_{t}(\mathbf{p}(0), \mathbf{q}(0)) / \hbar}|\mathbf{p}(t), \mathbf{q}(t)\rangle\langle\mathbf{p}(0), \mathbf{q}(0)|,
\end{aligned}
$$

where $(\mathbf{p}(t), \mathbf{q}(t))$ are the set of classically-evolved phase space coordinates, $S_{t}$ is the classical action and $C_{t}$ is a pre-exponential factor. ${ }^{24,35-46}$ In the Heller-Herman-KlukKay ${ }^{25,47}$ version of the SC-IVR, the pre-exponential factor,

$$
\begin{aligned}
& C_{t}(\mathbf{p}(0), \mathbf{q}(0)) \\
& =\sqrt{\frac{1}{2}\left|\frac{\partial \mathbf{q}(t)}{\partial \mathbf{q}(0)}+\frac{\partial \mathbf{p}(t)}{\partial \mathbf{p}(0)}-i \hbar \gamma \frac{\partial \mathbf{q}(t)}{\partial \mathbf{p}(0)}+\frac{i}{\gamma \hbar} \frac{\partial \mathbf{p}(t)}{\partial \mathbf{q}(0)}\right|}
\end{aligned}
$$

is given by the determinant of the combination of the four $F \times F$ size blocks of the $2 F \times 2 F$ symplectic (monodromy or stability) matrix $\mathbf{M}(t) \equiv \partial\left((\mathbf{p}(t), \mathbf{q}(t)) / \partial\left(\mathbf{p}_{0}, \mathbf{q}_{0}\right)\right)$. The accuracy of the classical propagation can be monitored by 
checking the deviation of the monodromy matrix determinant from unity. We adopted a restrict version of this check by monitoring the determinant of the positive-definite matrix $\mathbf{M}^{T} \mathbf{M}{ }^{48}$ In all simulations presented here, by adopting a time step of 10 a.u., the deviation were of the order of $10^{-5}$ in the worse case. Such a strict threshold will keep any spurious noisy signal order of magnitudes smaller than the vibrational peaks. The natural basis set representation of the semiclassical propagator in Eq. (5) is given by the direct product of one dimensional coherent states

$$
\begin{aligned}
\langle\mathbf{q} \mid \mathbf{p}(t), \mathbf{q}(t)\rangle= & \prod_{i}\left(\gamma_{i} / \pi\right)^{F / 4} \exp \left[-\frac{\gamma_{i}}{2} \cdot\left(q_{i}-q_{i}(t)\right)\right. \\
& \left.+\frac{i}{\hbar} p_{i}(t) \cdot\left(q_{i}-q_{i}(t)\right)\right],
\end{aligned}
$$

where $\gamma_{i}$ is fixed and equal to the width of the harmonic oscillator approximation to the wave function for each mode at the minimum of the PES where the dynamics will be carried out. Thus, we are using a diagonal width parameters matrix. Previous calculations have found the spectra to be relatively insensitive respect to significant changes in the width parameters, ${ }^{35}$ as shown in Fig. 4 of Ref. 20.

Thus, the SC-IVR approximation for the survival probability in Eq. (2) by the following Monte Carlo phase space integration:

$$
\begin{aligned}
\left\langle\chi\left|e^{-i \hat{H} t / \hbar}\right| \chi\right\rangle= & \frac{1}{(2 \pi \hbar)^{F}} \int d \mathbf{p}(0) \int d \mathbf{q}(0) \\
& \times C_{t}(\mathbf{p}(0), \mathbf{q}(0)) e^{i S_{t}(\mathbf{p}(0), \mathbf{q}(0)) / \hbar}\langle\chi \mid \mathbf{p}(t), \mathbf{q}(t)\rangle \\
& \times\langle\mathbf{p}(0), \mathbf{q}(0) \mid \chi\rangle,
\end{aligned}
$$

for any given reference state $|\chi\rangle=\left|\mathbf{p}_{e q}, \mathbf{q}_{e q}\right\rangle$. In this paper, we will examine the importance of the reference state choice and how one can enhance the spectral features by carefully choosing the reference state as a combination of coherent states. Our goal is to deal with many degrees of freedom, while keeping the computational effort low enough to allow for first principles semiclassical simulations.

Along this line, the time averaging filter was introduced. $^{32}$ In this SC-IVR implementation, the number of trajectories required for Monte Carlo integration are reduced at the cost of longer simulation times. ${ }^{33,34}$ The introduction of a time averaging integral is not an approximation per se: It does not imply any ergodicity property and it is in principles exact, by virtue of Liouville's theorem. The TA (time-averaging) SC-IVR expression for the spectral density is

$$
\begin{aligned}
I(E)= & \frac{1}{(2 \pi \hbar)^{F}} \int d \mathbf{p}(0) \int d \mathbf{q}(0) \frac{\mathrm{Re}}{\pi \hbar T} \int_{0}^{T} d t_{1} \\
& \times \int_{t_{1}}^{T} d t_{2} C_{t_{2}}\left(\mathbf{p}\left(t_{1}\right), \mathbf{q}\left(t_{1}\right)\right) \\
& \times\left\langle\chi \mid \mathbf{p}\left(t_{2}\right), \mathbf{q}\left(t_{2}\right)\right\rangle e^{i\left(S_{t_{2}}(\mathbf{p}(0), \mathbf{q}(0))+E t_{2}\right) / \hbar} \\
& \times\left[\left\langle\chi \mid \mathbf{p}\left(t_{1}\right), \mathbf{q}\left(t_{1}\right)\right\rangle e^{i\left(S_{t_{1}}(\mathbf{p}(0), \mathbf{q}(0))+E t_{1}\right) / \hbar}\right]^{*},
\end{aligned}
$$

where the sets $\left(\mathbf{p}\left(t_{1}\right), \mathbf{q}\left(t_{1}\right)\right)$ and $\left(\mathbf{p}\left(t_{2}\right), \mathbf{q}\left(t_{2}\right)\right)$ of position and momentum variables are the evolution of the initial phase space point $\left(\mathbf{p}_{0}, \mathbf{q}_{0}\right)$ at time $t_{1}$ and $t_{2}$ respectively, and $T$ is the total simulation time. The first time integration acts as the Fourier transform of Eq. (2) and the second is filtering the trajectories. This double-time integration is made by running any trajectory from time 0 to time $T$ and considering all possible intervals from $t_{1}$ and $t_{2}$. In this way, this integration considers all the trajectories that are generated as segments of time length $T-t_{1}$ out of a single long trajectory from time 0 to time $T$.

The most computational intense part of Eq. (9) is the calculation of the two times prefactor $C_{t_{2}}\left(\mathbf{p}\left(t_{1}\right), \mathbf{q}\left(t_{1}\right)\right)$ and its numerical stability greatly reduces during complex system simulations. This behavior can be tamed by adopting the separable approximation, where $C_{t_{2}}\left(\mathbf{p}\left(t_{1}\right), \mathbf{q}\left(t_{1}\right)\right)$ $\approx \exp \left[i\left(\phi\left(t_{2}\right)-\phi\left(t_{1}\right)\right) / \hbar\right]$ and $\phi(t)=\operatorname{phase}\left[C_{t}(\mathbf{p}(0), \mathbf{q}(0))\right]$. Then, Eq. (9) becomes

$$
\begin{aligned}
I(E)= & \frac{1}{(2 \pi \hbar)^{F}} \frac{1}{2 \pi \hbar T} \int d \mathbf{p}(0) \int d \mathbf{q}(0) \\
& \times \mid \int_{0}^{T} d t\langle\chi \mid \mathbf{p}(t), \mathbf{q}(t)\rangle \\
& \times\left. e^{i\left(S_{t}(\mathbf{p}(0), \mathbf{q}(0))+E t+\phi_{t}(\mathbf{p}(0), \mathbf{q}(0)) / \hbar\right)}\right|^{2},
\end{aligned}
$$

where now the double time integral is simplified to a single and positive-definite time integral. We found this approximation accurate and an order of magnitude less computational demanding.

\section{IMPLEMENTATION OF THE MULTIPLE COHERENT STATES SC-IVR FOR MOLECULAR VIBRATIONS}

The Monte Carlo phase space integration in Eq. (8) is usually performed with a number of trajectories of the order of thousands. ${ }^{35}$ This computation is quite intensive for first principles semiclassical dynamics calculations using state-ofthe-art ab initio methods. Recently, we developed the Multiple Coherent States SC-IVR (MC-SC-IVR) method ${ }^{21}$ which allowed us to significantly reduce the number of trajectories requested for the phase space integration to only a few, while still preserving good accuracy. The idea is pictorially represented in Fig. 1, where a schematic power spectrum is represented on panel (a) and the related potential on panel (b). On panel (c) the "eigen-trajectories," i.e., the classical trajectories with an energy corresponding to the location of the peaks, are represented in closed, continuous red lines. Several coherent states choices are reported with filled colored circles. The least useful choice of reference is represented in gray, i.e., with $|\chi\rangle=\left|\mathbf{p}_{e q}=0, \mathbf{q}_{e q}=0\right\rangle$. In this case, the Monte Carlo sampling is centered at the origin and the reference states will be mainly overlapped with trajectory points sampled in the harmonic region of the potential. From the appendix of Ref. 21 one can verify that for an harmonic oscillator of frequency $\omega$ this choice will result a power spectrum with a single ground-state peak $I(E)=\delta(E-\omega / 2)$. Eq. (1) suggests a better choice for the reference state is the state in which the 


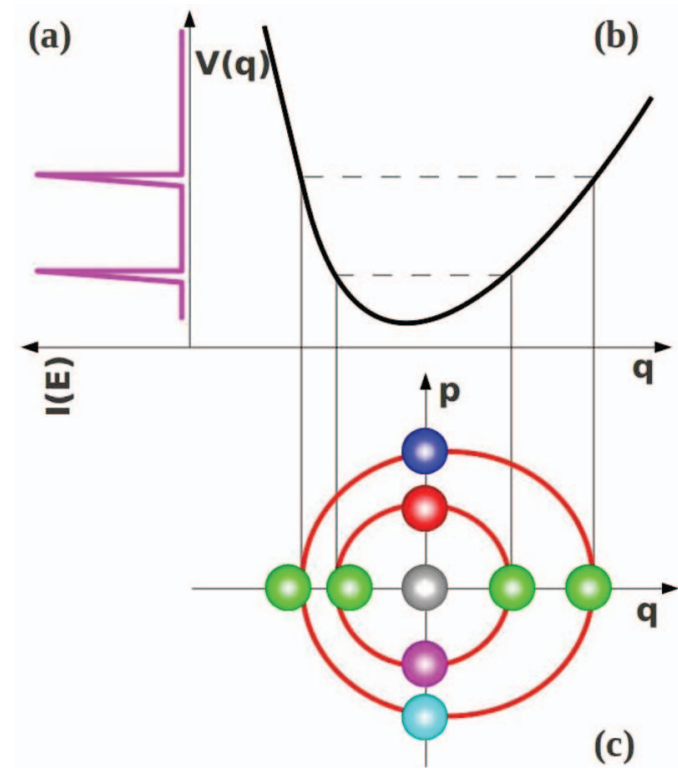

FIG. 1. Pictorial representation of several possible reference state representations for efficient multidimensional MC-SC-IVR simulation. On panel (a) the power spectrum; on panel (b) the potential energy and on panel (c) the phase space portrait where the classical trajectories (red closed lines) and the coherent states (filled colored circles) are reported.

overlap between the eigenfunctions and the reference states is maximum. This brought us to develop the Multiple Coherent States implementation of the SC-IVR, where the reference state is of the type

$$
|\chi\rangle=\sum_{i=1}^{N_{\text {states }}}\left|\mathbf{p}_{e q}^{i}, \mathbf{q}_{e q}^{i}\right\rangle
$$

and the phase space location of the state are reported in red and blue on Fig. 1, i.e., along the positive p-semi-axis. In this approach the coherent states are placed in a non-local fashion and kept fixed during the entire simulation. This is different from other semiclassical implementations where the coherent-state grid is updated to follow coherently the quantum wavepacket. ${ }^{49}$ One can equally choose the negative $\mathrm{p}$ semi-axis (the magenta and cyan states) or either the positive or the negative q-semi-axis (the green states). The location of the coherent states along the p-semi-axis has been determined in an harmonic fashion, namely, $p_{e q}^{2} / 2 m=\hbar \omega(n+1 / 2)$. One can run classical trajectories with these initial conditions, i.e., $\left(\mathbf{p}_{e q}, 0\right)$, and by checking the turning points he will calculate the location of the coherent state along the q-semi-axis. Such multiple coherent states methodology is supported by analytical investigations about the harmonic oscillator case, where for a generic reference state of the type $\left|p_{e q}, q_{e q}\right\rangle$, the power spectrum is fully determined by the sum over the integer index $k$

$$
\begin{aligned}
I(E)= & e^{-\left(p_{e q}^{2} / \hbar m \omega+q_{e q}^{2} m \omega / \hbar\right)} \sum_{k} \frac{1}{2^{k}}\left[\frac{p_{e q}^{2}}{\hbar m \omega}+\frac{m \omega q_{e q}^{2}}{\hbar}\right] \\
& \times \delta\left(E-\hbar \omega\left(k+\frac{1}{2}\right)\right),
\end{aligned}
$$

as one can deduce also from the Appendix of Ref. 21 by choosing $\gamma=m \omega / \hbar$. For the MC-SC-IVR method it was found that it is not crucial to know the exact location of the "eigen-trajectories". The Gaussian spreading of each coherent state lying on a trajectory chosen with initial harmonic conditions is generally wide enough to include the energy shells associated with the different energy peaks. This method allowed us to obtain accurate results for the $\mathrm{H}_{2} \mathrm{O}$ molecule on a model potential and for the $\mathrm{CO}_{2}$ molecule using an on-thefly approach. ${ }^{21}$ Using this method, we could also calculate the spectrum of a model potential describing the chemisorption process of $\mathrm{CO}$ on $\mathrm{Cu}(100)$ copper surface and the dipole spectra for a set of four dipole coupled $\mathrm{CO}$ molecules arranged in a realistic monolayer fashion. ${ }^{50}$ Finally, accurate vibrational eigenfunctions for the $\mathrm{CO}_{2}$ molecule were reproduced still on-the-fly. ${ }^{51}$

As mentioned in the introduction, in this manuscript, we strive to extend the MC-SC-IVR approach to deal with system with several degrees of freedom. As stressed in the introduction, useful simulations would be those where the recurrence overlap occurs on all dimensions simultaneously. This event is exponentially rare as the number of dimensions increases. A look at the survival probability expression in Eq. (8) in terms of positions and momenta for a single coherent state,

$$
\begin{aligned}
& \langle\chi \mid p(t), q(t)\rangle\langle p(0), q(0) \mid \chi\rangle \\
& =e^{-\gamma\left(q_{t}-q_{e q}\right)^{2} / 4} e^{-\gamma\left(q_{0}-q_{e q}\right)^{2} / 4-\left(p_{t}-p_{e q}\right)^{2} / 4 \hbar^{2} \gamma-\left(p_{0}-p_{e q}\right)^{2} / 4 \hbar^{2} \gamma} \\
& \quad \times e^{-i\left(p_{t} q_{t}-p_{0} q_{0}\right) / 2 \hbar-i p_{e q}\left(q_{t}-q_{0}\right) / 2 \hbar+i q_{e q}\left(p_{t}-p_{0}\right) / 2 \hbar}
\end{aligned}
$$

shows that whenever the trajectory occurs to be next to the $\left(p_{e q}, q_{e q}\right)$ coherent state center, the signal to be Fourier transformed is significant. The multidimensional semiclassical surviving probability of Eq. (4) is the product of several terms of the type of Eq. (13). Thus, the overlaps significantly occur only when, at the same time, each phase space set of coordinates is near to the center of the coherent state.

To improve the overlap for each trajectory, we suggest the strategic placement of coherent states along the periodic phase space trajectories, shown by the red continuous lines shown on Fig. 1. In other words, the reference state is given as an expression of the type of Eq. (11), where, for example, the phase space coordinates $\left(\mathbf{p}_{e q}, \mathbf{q}_{e q}\right)$ are located at the intersection of the eigen-trajectory with the phase space axes. In order to explore this idea, let us consider the spectrum of Eq. (9) where the reference state is a combination of the coherent states,

$$
\begin{aligned}
I(E)= & \frac{1}{(2 \pi \hbar)^{F}} \int d \mathbf{p}(0) \int d \mathbf{q}(0) \frac{\operatorname{Re}}{\pi \hbar T} \int_{0}^{T} d t_{1} \\
& \times \int_{t_{1}}^{T} d t_{2} C_{t_{2}}\left(\mathbf{p}\left(t_{1}\right), \mathbf{q}\left(t_{1}\right)\right)\left\langle\sum_{j} \mathbf{p}_{j}, \mathbf{q}_{j} \mid \mathbf{p}\left(t_{2}\right), \mathbf{q}\left(t_{2}\right)\right\rangle \\
& \times e^{i\left(S_{t_{2}}(\mathbf{p}(0), \mathbf{q}(0))+E t_{2}\right) / \hbar}\left[\left\langle\sum_{i} \mathbf{p}_{i}, \mathbf{q}_{i} \mid \mathbf{p}\left(t_{1}\right), \mathbf{q}\left(t_{1}\right)\right\rangle\right. \\
& \left.\times e^{i\left(S_{t_{1}}(\mathbf{p}(0), \mathbf{q}(0))+E t_{1}\right) / \hbar}\right]^{*}
\end{aligned}
$$


where $i$ and $j$ run independently over the combination in Eq. (11). In the Appendix, we show that for an harmonic oscillator, Eq. (14) becomes

$$
\begin{aligned}
I(E)= & \operatorname{Re} \sum_{i, j} e^{-m \omega\left(q_{i}^{2}+q_{j}^{2}\right) / 4 \hbar-\left(p_{i}^{2}+p_{j}^{2}\right) / 4 \hbar m \omega-i\left(p_{i} q_{i}-p_{j} q_{j}\right) / 2 \hbar} \\
& \times \sum_{k} \frac{1}{2^{k}}\left[\frac{p_{i} p_{j}}{\hbar m \omega}+\frac{m \omega q_{i} q_{j}}{\hbar}+\frac{q_{j} p_{i}-q_{i} p_{j}}{\hbar}\right]^{k} \\
& \times \delta\left(E-\hbar \omega\left(k+\frac{1}{2}\right)\right) .
\end{aligned}
$$

If we consider for this case a reference state of the type $|\chi\rangle_{p}=|p, 0\rangle+|-p, 0\rangle$ (for example the red and magenta states on Fig. 1), where the subscript $p$ indicates that the states are along the p-axes), the resulting power spectrum will be

$$
I(E)=2 e^{-p^{2} / 2 \hbar m \omega} \sum_{k E V E N} \frac{p^{2 k}}{2^{k}(\hbar m \omega)^{k}} \delta\left(E-\hbar \omega\left(k+\frac{1}{2}\right)\right),
$$

and all the even peaks of the harmonic spectra will be calculated. Instead, if the antisymmetric combination $|\chi\rangle_{p}$ $=|p, 0\rangle-|-p, 0\rangle$ would have been chosen, one will obtain a sum over all odd peaks. The same reasoning is valid for the green coherent state along the q-axes in Fig. 1. Instead, mixed choices of the type $|\chi\rangle=|p, 0\rangle \pm|0, \pm q\rangle$ will not be able to selectively produce odd or even power spectra peaks, but the entire spectrum. Eventually, one can cleverly choose the values of the phase space coordinates of each coherent state in a way to select or project different peaks. For example, in a multidimensional case, one can selectively make antisymmetric just a single mode in order to identify the peak corresponding to odd excitations of that mode. We will show some examples below. At the same time, we will obtain more recurring overlapping events because of the different product combinations between coherent states.

Finally, we will show that also in the case of a reference state of the type,

$$
|\chi\rangle_{p q}=|p, 0\rangle \pm|0, q\rangle \pm|-p, 0\rangle \pm|0,-q\rangle
$$

(where the $p q$ subscript indicates the choice of 4 coherent states, i.e., each one per semi-axes), there are suitable combinations for the selection of peaks. Clearly, for a reference state of the type of Eq. (17), the recurrence events are even more frequent. For the harmonic oscillator, one will find that only some combinations will allow the selection of peaks. These results are reported in Table I. Such a tool for selecting power spectrum peaks will result extremely convenient for multidimensional spectra where the identification of peaks could become an intractable problem by itself.

\section{THE MANY-DIMENSIONAL MORSE SYSTEM: AN ANALYTICAL TEST OF THE PROPOSED APPROACH}

In order to apply the idea introduced in the previous section, we explore the example of two uncoupled Morse po-
TABLE I. Analytical power spectra for an harmonic oscillator using Eq. (14) for a reference state of the type $|\chi\rangle=|p, 0\rangle+a|0, q\rangle$ $+b|-p, 0\rangle+c|0,-q\rangle$. The results are expressed in terms of $P=e^{-p^{2} / 2 \hbar m \omega}$ $\times \sum_{k} p^{2 k} \delta(E-\hbar \omega(k+1 / 2)) /(\hbar m \omega)^{k} 2^{k}, \quad Q=e^{-m \omega q^{2} / 2 \hbar} \sum_{k} q^{2 k}(m \omega)^{k} \delta$ $\times(E-\hbar \omega(k+1 / 2)) /(2 \hbar)^{k}$ and $P Q=e^{-p^{2} / 4 \hbar m \omega-m \omega q^{2} / 4 \hbar} \sum_{k}(q p)^{k} \delta(E$ $-\hbar \omega(k+1 / 2)) /(2 \hbar)^{k}$.

\begin{tabular}{lcc}
\hline \hline a,b,c). & $\mathrm{I}(\mathrm{E})$. & Parity \\
\hline$(+1,+1,+1)$. & $2\left(1+(-1)^{k}\right)(P+Q+2 P Q)$. & even \\
$(-1,+1,+1)$. & $2 P\left(1+(-1)^{k}\right)+2 Q\left(1-(-1)^{k}\right)$. & none \\
$(+1,-1,+1)$. & $2 P\left(1-(-1)^{k}\right)+2 Q\left(1+(-1)^{k}\right)$. & none \\
$(+1,+1,-1)$. & $2 P\left(1+(-1)^{k}\right)+2 Q\left(1-(-1)^{k}\right)$. & none \\
$(-1,-1,+1)$. & $2\left(1-(-1)^{k}\right)(P+Q)$. & odd \\
$(+1,-1,-1)$. & $2\left(1-(-1)^{k}\right)(P+Q)$. & odd \\
$(-1,+1,-1)$. & $2\left(1+(-1)^{k}\right)(P+Q+2 P Q)$. & even \\
$(-1,-1,-1)$. & $2 P\left(1-(-1)^{k}\right)+2 Q\left(1+(-1)^{k}\right)$. & none \\
\hline \hline
\end{tabular}

tentials. The oscillator frequencies have been chosen in a way to avoid degeneracy of the overtones. The standard procedure would be that one of using the reference state $|\chi\rangle$ $=\left|p_{1}, q_{1}\right\rangle\left|p_{2}, q_{2}\right\rangle$ for Eq. (9). For the purposes of this example, we have used the single-trajectory approximation of 3000 time-steps of 10 a.u. each, and the separable approximation in Eq. (10) to obtained the spectrum shown in black in Fig. 2. This reference state has no symmetry restrictions and all peaks are represented by the black line. Following, we employed the same trajectory but using the reference state $|\chi\rangle_{p}$ $=\left(\left|p_{1}, q_{1}\right\rangle+\left|-p_{1}, q_{1}\right\rangle\right)\left(\left|p_{2}, q_{2}\right\rangle+\left|-p_{2}, q_{2}\right\rangle\right)$, where $\left(p_{1}\right.$, $q_{1}$ ) can be chosen at the location of the red coherent state on Fig. 1 and $\left(p_{2}, q_{2}\right)$ at the location of the magenta coherent state. This results in two coherent states placed along the paxes but with opposite momenta. In this way, we were able to select all the even peaks of both oscillators. This choice is represented with the red line shown in Fig. 2 and the peak labels of $(0,0),(0,2)$ and $(2,0)$. One can select the odd excitations of the first Morse oscillator by choosing the combination $|\chi\rangle_{p}$ $=\left(\left|p_{1}, q_{1}\right\rangle-\left|-p_{1}, q_{1}\right\rangle\right)\left(\left|p_{2}, q_{2}\right\rangle+\left|-p_{2}, q_{2}\right\rangle\right)$ and of the

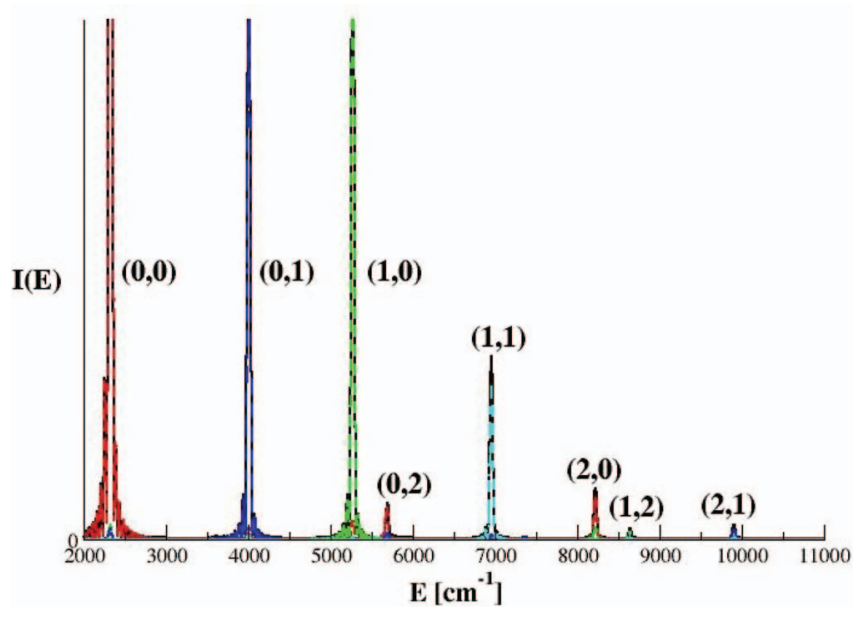

FIG. 2. Power spectrum (black line) of two uncoupled Morse potentials with the following parameters: dissociation energy $D_{e}=0.2$ a.u., unitary masses, $\omega_{1}=3000 \mathrm{~cm}^{-1}$ and $\omega_{2}=1700 \mathrm{~cm}^{-1}$. The red line represents all the even peaks, blue represents the odd excitations on the first oscillator, while green is used for the odd excitations on the second oscillator. Finally, cyan is employed for the simultaneous odd excitations on both oscillators. 
TABLE II. Frequencies values for the 19-dimensional Morse system. MO stands for Morse Oscillator. $\mathrm{ZPE}_{H}$ is the Harmonic Zero Point Energy, while $\mathrm{ZPE}$ is the analytical one. All data in $\mathrm{cm}^{-1}$.

\begin{tabular}{lccccr}
\hline \hline MO & Freq. & MO & Freq. & MO & Freq. \\
\hline 1 & 3095 & 8 & 1297 & 15 & 969 \\
2 & 3080 & 9 & 1183 & 16 & 843 \\
3 & 3061 & 10 & 1162 & 17 & 701 \\
4 & 3051 & 11 & 1036 & 18 & 607 \\
5 & 1607 & 12 & 997 & 19 & 402 \\
6 & 1483 & 13 & 996 & ZPE $_{H}$ & 13959 \\
7 & 1365 & 14 & 983 & ZPE & 13880 \\
\hline \hline
\end{tabular}

second one with the combination $|\chi\rangle_{p}=\left(\left|p_{1}, q_{1}\right\rangle+\mid-p_{1}\right.$, $\left.\left.q_{1}\right\rangle\right)\left(\left|p_{2}, q_{2}\right\rangle-\left|-p_{2}, q_{2}\right\rangle\right)$. These choices are represented respectively by the green continuous line (the $(1,0)$ peak) and in blue (the $(0,1)$ peak). Finally, one can force both modes to be antisymmetric at the same time with the reference state $|\chi\rangle_{p}$ $=\left(\left|p_{1}, q_{1}\right\rangle-\left|-p_{1}, q_{1}\right\rangle\right)\left(\left|p_{2}, q_{2}\right\rangle-\left|-p_{2}, q_{2}\right\rangle\right)$. This choice is reported by the cyan line in Fig. 2 and the $(1,1)$ label.

This example sets the stage for more realistic multidimensional tests, where the advantages of this coherent-states emerge. We considered a system of 19 Morse oscillators, where the frequency of each oscillator is reported in Table II. Such a set of frequency values are the benzene normal modes ones after removing the redundant degeneracies. By using this choice, the set of vibrations is quite realistic and ranges from the high-energy stretching modes to the floppy ring torsional modes. In order to show the full potential of the multiple coherent state approach, we consider a simulation employing a single trajectory. On panel (a) of Fig. 3 the power spectrum for a single coherent state is reported in a continuous black line. This spectrum is very noisy and one cannot even un-

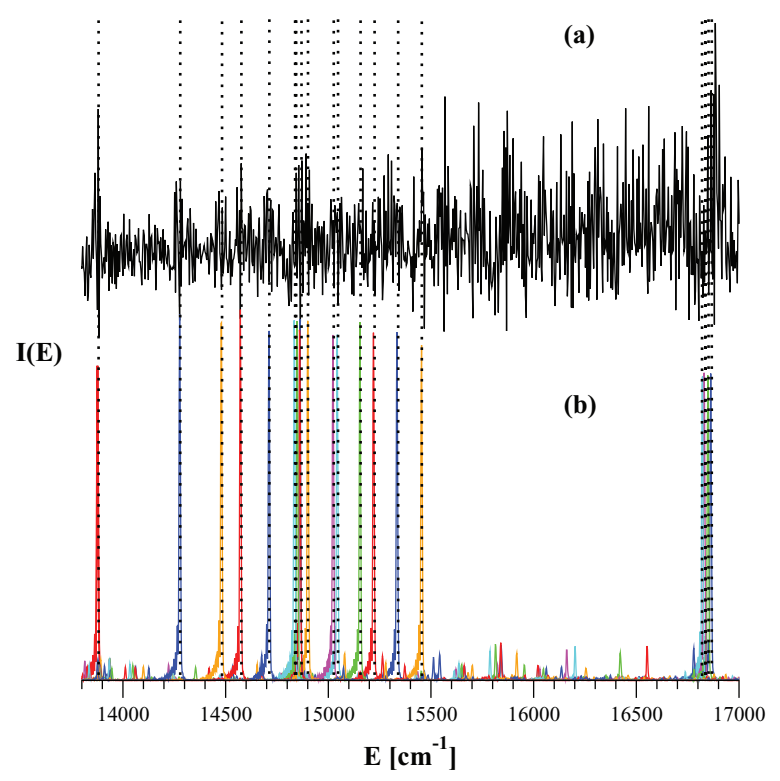

FIG. 3. Power spectrum of 19 uncoupled Morse oscillators of unitary masses and dissociation energy $D_{e}=0.2$ a.u.. Each oscillator frequency are reported in Table II. All lines are obtained with a single trajectory simulation: Panel (a) and black line for a single coherent state; Panel (b) and colored lines for the two coherent states MC-SC-IVR implementation. The vertical dashed lines represent the exact values of all possible single excitations. equivocally identify the zero point energy (ZPE) peak. This is problematic since from previous experience, we have always found it on systems of smaller dimensionality by means of a single trajectory. ${ }^{20,32} \mathrm{We}$ also find the counter-intuitive result that the spectrum is more intense for the higher energy range. This is in contradiction with the fact the reference state is located at the harmonic ZPE position and the trajectory is also within such energy shell. This requires further study.

A clear improvement is obtained when the multiple coherent state formulation of the reference state described above is adopted. As an example, the reference state was set as the combination of two coherent states located as the red and the magenta points in Fig. 1. By taking the symmetrical combination and using the same classical trajectory of panel (a), we obtained the spectrum on panel (b) with the ZPE peak shown in red. Then, by imposing the antisymmetric combination to each mode one by one, we generated the series of singleexcitation peaks reported with different colors on the same panel of Fig. 3. The accuracy of these peaks is demonstrated by the vertical dashed lines, which are the analytical values of the vibrational energies of the 19-dimensional Morse system. In the upper spectrum of Fig. 3 there are several noisepeaks between the exact eigenvalues shown by vertical dashed lines. These peaks are unphysical and they do not relate to any eigenvalue. Instead, in the lower panel, the favorable statistics generated by the combination of coherent states greatly reduced the intensity of these peaks and enhanced the eigenvalues peaks, that now are clearly distinguishable. Eventually, we have not only reproduced the power spectrum of this Morse system but also distinguished and determined each peak. This is an important advantage for calculating less obvious spectra. Finally, by using this uncoupled oscillators model, we didn't want to test the ability of the MC-SC-IVR to reproduce mode couplings effects. Our purpose was to overcome the statistical issue related exclusively to the increase of dimensionality. On the other side, the MC-SC-IVR has already been successfully tested on anharmonic and strongly coupled systems, such as the $\mathrm{CO}_{2}$ and $\mathrm{H}_{2} \mathrm{O}$ molecules. In Sec. V, we extend the method to the first-principles calculation of a molecule with dimensions larger than those of $\mathrm{H}_{2} \mathrm{O}$.

\section{FIRST PRINCIPLES SEMICLASSICAL MOLECULAR DYNAMICS OF FORMALDEHYDE}

In a very recent paper Roy et $a .^{23}$ performed $a b$ initio semiclassical simulation of the formaldehyde molecule, using SC-IVR methodology. ${ }^{44}$ This simulation provides a good benchmark for different first-principles semiclassical molecular dynamics approaches. The formaldehyde molecule is a well tested system, in particular, at the semiclassical and vibrational configuration interaction (VCI) level. ${ }^{32,44}$ In the VCI method, a variational self consistent field (VSCF) procedure is adopted to find the solution of the vibrational Schroedinger equation. ${ }^{52,53}$ The ansatz for the vibrational wavefunction is given by the direct product of unidimensional functions. The application of the variational principle to the eigenstates equation for this ansatz brings a set of equations coupled through a mean-field potential for each unidimensional eigenfunction. The correlated 

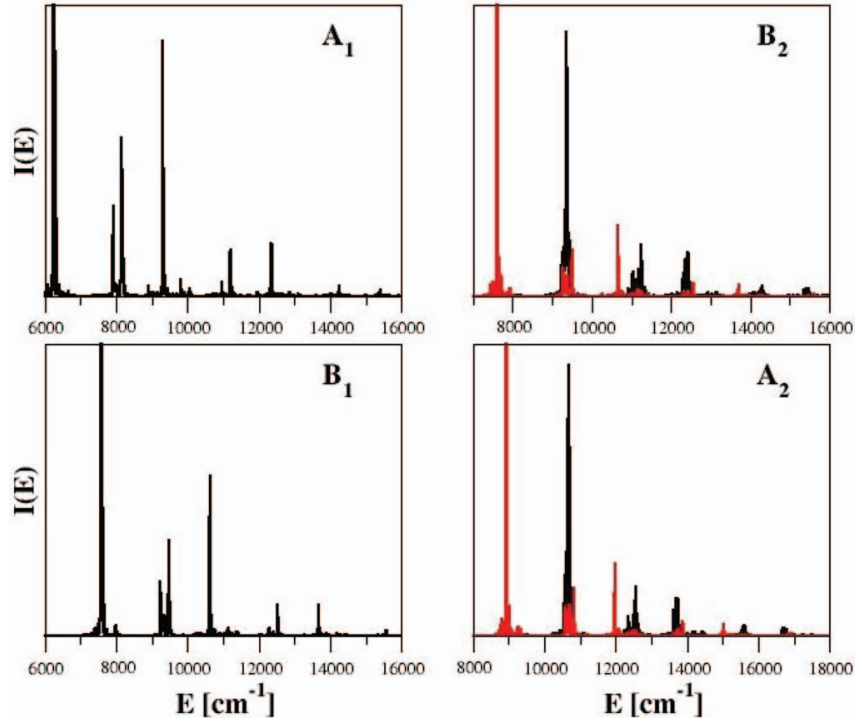

FIG. 4. First principles MC-TA-SC-IVR spectrum calculations for formaldehyde using the separable approximation of Eq. (10). Data have been separated according to the irreducible representations of the $C_{2 V}$ point group of the molecule. On panel $A_{1}$ all coherent state coefficients $\lambda$ are set to 1 . On panel $\mathrm{B}_{2}$, the red (black) line corresponds to the combination $\lambda_{2}=-1\left(\lambda_{6}=-1\right)$ and all others set to 1 . On panel $\mathrm{B}_{1}, \lambda_{1}=-1$ and on panel $\mathrm{A}_{2}$ the red (black) line corresponds to $\lambda_{1}=\lambda_{2}=-1\left(\lambda_{1}=\lambda_{6}=-1\right)$ and all others set to 1 .

vibrational eigenstates are calculated by diagonalizing the full Hamiltonian in a virtual VSCF basis. ${ }^{54-56}$

In order to have a full comparison with the previously reported simulations, we have performed electronic structure calculations at the same (quite modest) HF/3-21G level of theory. The equilibrium geometry and the vibrational frequencies of Ref. 23 were faithfully reproduced using the Q-Chem electronic structure package..$^{57}$ We ran a set of 5,000 timesteps (of 10 a.u. time step) trajectories. The power spectra using the MC-TA-SC-IVR method are reported in Fig. 4. These have been divided into four panels according to the respective $C_{2 V}$ irreducible representations. In order to select the peaks in this fashion, we implemented coherent state combination of Eq. (11). The more comprehensive and general implementation includes first the coherent states with opposite momenta, which are added to Eq. (11),

$$
|\chi\rangle=\sum_{k=1}^{N_{\text {states }}}\left|\mathbf{p}_{e q}^{k}, \mathbf{q}_{e q}^{k}\right\rangle+\varepsilon_{k}\left|-\mathbf{p}_{e q}^{k}, \mathbf{q}_{e q}^{k}\right\rangle
$$

where, for each $k, \varepsilon_{k}$ is a vector of $F$ dimension (the number of degrees of freedom) and its components are equal to \pm 1 . This set up for the reference states allowed us to take all the advantages described for the Morse systems reported above. Then, in order to restrict the peaks to a given symmetry space irreducible representation, each state in each dimension is further written in terms of a combination of the type

$$
\left|p_{e q, i}^{(k)}, q_{e q, i}^{(k)}\right|^{\lambda_{k, i}}=\left(\left|p_{e q, i}^{(k)}, q_{e q, i}^{(k)}\right\rangle+\lambda_{k, i}\left|-p_{e q, i},-q_{e q, i}^{(k)}\right|\right) / \sqrt{2} .
$$

In this way, the $\mathrm{i}$-th mode component can be made symmetric $\left(\lambda_{k}=1\right)$ or antisymmetric $\left(\lambda_{k}=-1\right)$. In conclusion, the reference state representation can be more concisely be written as

$$
|\chi\rangle=\sum_{k=1}^{N_{\text {states }}} \prod_{j=1}^{F} \varepsilon_{k}(j)\left|p_{e q, j}^{k}, q_{e q, j}^{k}\right\rangle,
$$

where $j$ runs over the degrees of freedom, and $k$ over all coherent states. The original number of MC-SC-IVR states are therefore duplicated by changing the momenta sign as in Eq. (18) and all these states further duplicated by changing both the momenta and positions sign as in Eq. (19). The use of symmetry by means of the describe strategy allows for a substantial reduction of the Hilbert space sampled, and therefore results in an increase of the amplitudes of the desired states.

To obtain the spectra in Fig. 4, we didn't use terms of the type of Eq. (18), but only of the type of Eq. (19). Instead, the spectra in Fig. 5 have been calculated by using the combinations of the type in Eq. (18) and without symmetry restrictions. The results shown in Fig. 5 are neat and the peaks are very well centered. Noise has been reduced and the peaks were selected by using reference states of the type of Eq. (18). The full spectrum is reported on the panels $a$ and $a^{\prime \prime}$. On the left part, i.e., from spectrum $b$ to $g$, the single excitations spectra are calculated for each of the six vibrational normal modes of the formaldehyde molecule. On the right part, the double excitation peaks are reported: Spectrum $b^{\prime \prime}$ is for the antisymmetric combination, i.e., $\varepsilon=-1$, on the first and second mode; spectrum $c^{\prime \prime}$ on the first and the third, spectrum $d^{\prime \prime}$ on the first and the fourth; spectrum $e^{\prime \prime}$ on the second and the third; spectrum $f^{\prime \prime}$ on the second and the fourth and finally spectrum $g^{\prime \prime}$ is obtained by placing the odd combination on the third and the fourth mode. In these illustrative calculations we always used the same ground state trajectory but a different reference state set-up. Accuracy can be added by varying the initial trajectory condition according to the

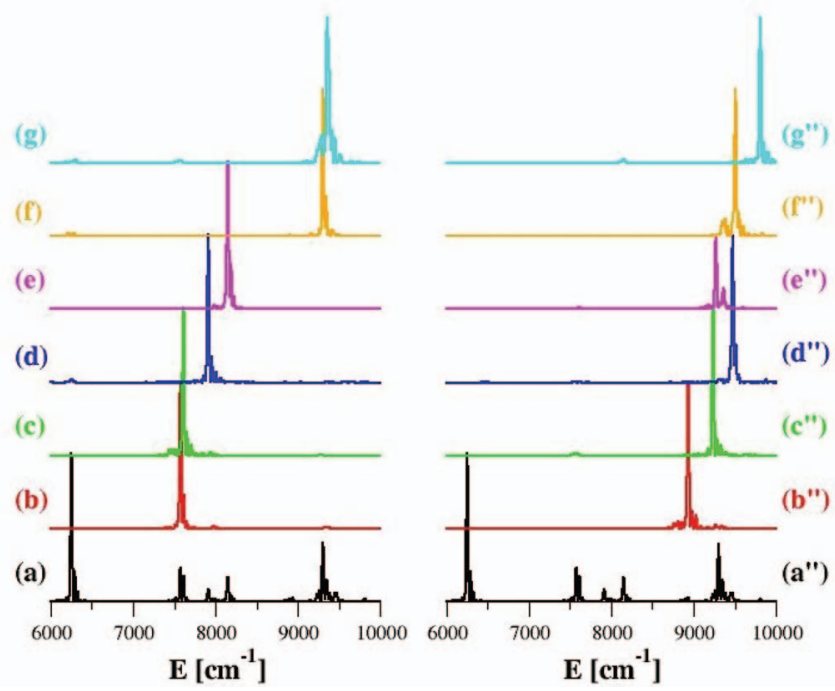

FIG. 5. First principles single trajectory power spectra of formaldehyde with different reference state formulations. On panel (a) and (a") the full spectrum. On the left part (b to g), single excitation peaks are reported, while on the right ( $\mathrm{b}^{\prime \prime}$ to $\mathrm{g}^{\prime \prime}$ ) combinations of single excitations for the lower four modes. See main text for each peak denomination. 
TABLE III. First principles MC-TA-SC-IVR $A_{1}$ symmetry vibrational energy levels. In the first column, the state is denoted by the number of the excited mode and its quanta of excitation as subscript. The second column the harmonic estimate are reported. On the following columns, the MC-TA-SC-IVR method is employed (with or without the separable approximation indicated by "/S") either with a single trajectory or with the eight trajectories corresponding to the first eight excited stated.

\begin{tabular}{|c|c|c|c|c|c|c|c|c|c|c|c|}
\hline State & Harm. & $\mathrm{MC}[1] / \mathrm{S}$ & $\mathrm{MC}[1]$ & $\mathrm{MC}[8] / \mathrm{S}$ & $\mathrm{MC}[8]$ & State & Harm. & $\mathrm{MC}[1] / \mathrm{S}$ & $\mathrm{MC}[1]$ & $\mathrm{MC}[8] / \mathrm{S}$ & $\mathrm{MC}[8]$ \\
\hline ZPE & 6360 & 6256 & 6256 & 6258 & 6257 & $4_{1} 5_{1}$ & 11438 & 11202 & 11199 & 11163 & $11167^{\mathrm{a}}$ \\
\hline $3_{1}$ & 8053 & 7915 & 7914 & 7911 & 7939 & $3_{3}$ & 11438 & 11239 & $11238^{a}$ & 11203 & 11202 \\
\hline $4_{1}$ & 8276 & 8152 & 8153 & 8152 & 8161 & $3_{2} 4_{1}$ & 11661 & 11472 & $11473^{a}$ & 11451 & 11483 \\
\hline $1_{2}$ & 9034 & 8904 & 8903 & 8845 & 8847 & $3_{1} 4_{2}$ & 11884 & 11704 & $11708^{a}$ & 11673 & 11673 \\
\hline $2_{2}$ & 9116 & 8969 & 8969 & 8896 & 8885 & $4_{3}$ & 12107 & 11951 & 11951 & 11905 & 11907 \\
\hline $5_{1}$ & 9522 & 9302 & 9303 & 9300 & 9303 & $2{ }_{2} 3_{2}$ & 12502 & 12236 & $12231^{\mathrm{a}}$ & 12293 & 12260 \\
\hline $3_{2}$ & 9745 & 9583 & $9584^{\mathrm{a}}$ & 9598 & 9597 & $5_{2}$ & 12685 & 12355 & 12350 & 12352 & 12355 \\
\hline $3_{1} 4_{1}$ & 9968 & 9810 & 9808 & 9819 & 9819 & $6_{2}$ & 12826 & 12523 & 12474 & 12419 & 12507 \\
\hline $4_{2}$ & 10191 & 10050 & 10054 & 10020 & 10020 & $3_{2} 5_{1}$ & 12908 & 12625 & $12618^{\mathrm{a}}$ & 12599 & 12601 \\
\hline $1_{2} 3_{1}$ & 10727 & 10556 & 10556 & 10537 & 10487 & $3_{4}$ & 13131 & 12862 & 12861 & 12829 & 12830 \\
\hline $2{ }_{2} 3_{1}$ & 10809 & 10663 & $10649^{\mathrm{a}}$ & 10629 & 10578 & $4_{2} 5_{1}$ & 13354 & 13102 & 13103 & 13041 & 13043 \\
\hline $1_{2} 4_{1}$ & 10950 & 10719 & $10708^{a}$ & 10730 & 10732 & $3_{1} 5_{2}$ & 14377 & 14014 & 14013 & 13925 & 13919 \\
\hline $26_{1}$ & 10971 & 10795 & 10795 & 10775 & $10797^{\mathrm{a}}$ & $4_{1} 5_{2}$ & 14600 & 14246 & 14247 & 14181 & 14180 \\
\hline $3_{1} 5_{1}$ & 11215 & 10962 & 10965 & 10942 & 10944 & $3_{2} 5_{2}$ & 16070 & 15401 & 15401 & 15401 & 15404 \\
\hline
\end{tabular}

${ }^{\mathrm{a}}$ Uncertain peak.

vibrational state energy shell of the selected peak. Otherwise, one can use the MC-SC-IVR method where a set of trajectories, each one starting from each coherent state, is employed at the same time. When these results are restricted to the $A_{1}$ symmetry for the same vibrational states of Ref. 23, we obtained the vibrational values reported in Table III. On the same Table, the vibrational states are denoted by the excited mode and the subscript indicates the number of quanta of that particular mode. For example, the notation $3_{2} 4_{1}$ means that the third mode has been double excited, while the fourth single excited. The modes are numbered starting from the lower frequency one. On the second column the harmonic vibrational energy levels are reported. In the following, two columns single trajectories results are presented, with (denoted by "S") or without the separable approximation. In the last two columns, MC-TA-SC-IVR results with or without the separable approximation are reported. The multiple coherent states method employed the eight lower vibrational energy eigen-trajectories. As a rule of thumb, we have noticed that MC-TA-SC-IVR results are more anharmonic than single trajectory runs, especially for higher vibrational states. Then, no significant difference has been observed between the set of vibrational values calculated using the separable approximation and without it, even if this last type of spectra are more noisy.

Finally, the comparison between our calculations and the previous ones on the same $a b$ initio set up is reported in Table IV. Here different VCI approaches results are also reported directly from Ref. 23 in conjunction with their semiclassical ones. We can denote an overall agreement between the different methods. We have chosen to report in Table IV the vibrational energies calculated at the level of $\mathrm{MC}[8] / \mathrm{S}$ theory, because this showed less uncertain peaks. Looking at Table IV in more details, one can see how the MC[8]/S is fairly in agreement with the correlation-corrected vibrational self-consistent field/two-mode coupling representation of a quartic force field (cc-VSCF/2MR-QFF) results, the direct correlation corrected VSCF ones and the direct rectilinear vibrational self-consistent field/vibrational configuration interaction with perturbation selected interactions-second-order perturbation theory (VSCF/VCIPSI-PT2) ones. At lower energy the MC-TA-SC-IVR method is including more anharmonic contributions than the VCI family of results, while at higher energies is the opposite. On the other side, the semiclassical results of Roy et ll. $^{23}$ agree better with the last VCI column, i.e., the C-VSCF/VCIPSI-PT2. In their calculations Roy et al. ${ }^{23}$ do not use the time-averaging filter and used the Johnson multichannel WKB approximation ${ }^{58,59}$ for the prefactor of Eq. (6). Then, they simulate directly in Cartesian coordinates and not in normal mode ones, as we did. For these reasons, better agreement of the MC-SC-IVR method with the VSCF than VCI may be due to the choice of common normal modes coordinates. ${ }^{60}$ The Cartesian coordinates allow them to properly include kinetic cross terms. ${ }^{61-64}$ In their first principles simulations, 20000 trajectories of 244 fs simulation time each were employed. Even if a certain percentage of these trajectories is discarded according to the spectra energy window of interest, this is clearly an intensive calculation because the Hessian must be evaluated at each time-step. In order to enhance the spectral accuracy, they changed the reference state according to the energy window, i.e., to the vibrational state of interested as introduced by us before. ${ }^{21}$ Their coherent states are placed along the coordinates axes, while keeping the momenta equal to zero, as suggested by the gray filled circles in Fig. 1 of Ref. 21.

In conclusion, the two ab initio semiclassical simulations compared in this work have several common features, but still the semiclassical propagator is too different to ascribe any discordance in the results to a specific choice in the propagator representation. Nevertheless, we consider the two simulations in fair agreement. 
TABLE IV. Formaldehyde $\mathrm{A}_{1}$ symmetry power spectrum. Second column the harmonic approximation; on the third the correlation-corrected vibrational selfconsistent field/two-mode coupling representation of a quartic force field results; on the fourth the direct correlation corrected VSCF ones; on the fifth the direct rectilinear vibrational self-consistent field/vibrational configuration interaction with perturbation selected interactions-second-order perturbation theory; on the sixth the direct curvilinear; on the last the MC-SC-IVR.

\begin{tabular}{|c|c|c|c|c|c|c|c|}
\hline State & Harm. & $\begin{array}{c}\mathrm{cc}-\mathrm{VSCF} / \\
2 \mathrm{MR}-\mathrm{QFF}^{44}\end{array}$ & $\begin{array}{c}\text { Direct } \\
\text { cc-VSCF }\end{array}$ & $\begin{array}{c}\text { VSCF/ } \\
\text { VCIPSI-PT2 }{ }^{44}\end{array}$ & $\begin{array}{c}\text { C-VSCF } \\
\text { VCIPSI-PT2 } 2^{44}\end{array}$ & Wong et al. ${ }^{23}$ & $\begin{array}{c}\mathrm{MC}^{8} / \\
\mathrm{S}\end{array}$ \\
\hline $\mathrm{ZPE}$ & 6360 & 6271 & 6268 & 6268 & 6309 & 6311 & 6258 \\
\hline $3_{1}$ & 8053 & 7924 & 7922 & 7921 & 7980 & 8013 & 7911 \\
\hline $4_{1}$ & 8276 & 8155 & 8152 & 8152 & 8198 & 8208 & 8152 \\
\hline $5_{1}$ & 9522 & 9265 & 9254 & 9251 & 9320 & 9303 & 9300 \\
\hline $3_{2}$ & 9745 & 9574 & 9571 & 9571 & 9650 & 9587 & 9598 \\
\hline $34_{1}$ & 9968 & 9801 & 9805 & 9794 & 9858 & 9858 & 9819 \\
\hline $4_{2}$ & 10191 & 10029 & 10024 & 10023 & 10074 & 10089 & 10020 \\
\hline $3_{1} 5_{1}$ & 11215 & 10913 & 10885 & 10876 & 10981 & 10896 & 10942 \\
\hline $4_{1} 5_{1}$ & 11438 & 11164 & 11133 & 11126 & 11211 & 11191 & 11163 \\
\hline $3_{3}$ & 11438 & 11164 & 11216 & 11216 & 11321 & 11269 & 11203 \\
\hline $3_{2} 4_{1}$ & 11661 & 11439 & 11453 & 11432 & 11517 & 11562 & 11451 \\
\hline $3_{1} 4_{1}$ & 11884 & 11665 & 11672 & 11653 & 11722 & 11690 & 11673 \\
\hline $4_{3}$ & 12107 & 11893 & 11885 & 11881 & 11936 & 11961 & 11905 \\
\hline $5_{2}$ & 12685 & 12183 & 12136 & 12259 & 12232 & 12297 & 12352 \\
\hline $3_{2} 5_{1}$ & 12908 & 12525 & 12511 & 12498 & 12642 & $12488^{\mathrm{a}}$ & 12599 \\
\hline $3_{4}$ & 13131 & $\ldots$ & 12851 & 12851 & 12986 & 12904 & 12829 \\
\hline $4_{2} 5_{1}$ & 13354 & 13025 & 12997 & 12987 & 13088 & 13086 & 13041 \\
\hline $3_{2} 4_{2}$ & 13576 & $\ldots$ & 13316 & 13279 & 13370 & $13544^{\mathrm{a}}$ & 13344 \\
\hline $4_{4}$ & 14022 & $\ldots$ & 13735 & 13727 & 13787 & 13853 & 13767 \\
\hline $3_{1} 5_{2}$ & 14377 & 13795 & 13735 & 13844 & 13884 & 14115 & 13925 \\
\hline $4_{1} 5_{2}$ & 14600 & 14072 & 14006 & 14120 & 14126 & $14055^{\mathrm{a}}$ & 14181 \\
\hline $5_{3}$ & 15847 & 15071 & 14955 & 15103 & 15254 & 15209 & 15271 \\
\hline $3_{2} 5_{2}$ & 16070 & $\ldots$ & 15329 & 15430 & 15533 & $15591^{\mathrm{a}}$ & 15401 \\
\hline $4_{2} 5_{2}$ & 16516 & $\ldots$ & 15960 & 15967 & 16004 & 15952 & $15832^{\mathrm{a}}$ \\
\hline
\end{tabular}

${ }^{\mathrm{a} U n c e r t a i n}$ peaks.

\section{CONCLUSIONS}

Complex systems are still out of reach for a first principles approach of quantum dynamics. There can be several factors that hamper semiclassical methods for this kind of applications, since a system complexity is induced not only by coupling, strong anharmonic effects or multiple local PES minima, but also it is originated by the inherent "curse of dimensionality" associated with functional integration on classical computers. In this work, we focused on this last issue and developed a strategy that allowed us to tackle this problem with a first principles semiclassical approach, i.e., using only few classical trajectories. To reach such a goal, first the dimensionality semiclassical limitations have been analyzed analytically and by model systems. Then, our recently developed MC-SC-IVR method has been implemented in a way to overcome these limitations, using the same spirit that animated its original developments. Multiple coherent states are placed along the phase space trajectory. In this way, we obtain a two-fold improvement: favorable statistic is enhanced and peaks can be selected. Finally, first principles MC-SCIVR simulations have been presented for the formaldehyde molecule and results are compared with recent studies performed with similar methods. ${ }^{23}$

Then, the use of coherent states presented in Secs. III and IV can easily be extended to thousands SC-IVR trajectory simulations. Instead of writing the reference state as a single ground coherent state of the type $|\chi\rangle=\left|\mathbf{p}_{e q}, \mathbf{q}_{e q}\right\rangle$, one just needs to represent it as a two states combination of the type $|\chi\rangle=\left|\mathbf{p}_{e q}, \mathbf{q}_{e q}\right\rangle \pm\left|-\mathbf{p}_{e q}, \mathbf{q}_{e q}\right\rangle$. In this case, the peak intensities are still proportional to the transition probability from the ground state population when, for example, a dipole absorption spectrum formulation is adopted, and the implementation takes just few lines of code. As far as the Monte Carlo phase space sampling is concerned, the Husimi distribution must be accordingly modified.

In conclusion, we hope that in the future realistic higher dimensional systems can be tackled on-the-fly with this MCSC-VR implementation.

\section{ACKNOWLEDGMENTS}

A. Brown and P.-N. Roy are thanked for useful discussions. The University of Milan is thanked for funding (PUR grant) and CILEA (Consorzio Interuniversitario Lombardo per l'Elaborazione Automatica) for computational time allocation. Authors thank also FAS Research Computing for computational support. A.A.-G. acknowledges support from the Sloan Foundation and the Camille and Henry Dreyfus foundation.

\section{APPENDIX: NON-LOCAL REFERENCE STATES SPECTRA FOR HARMONIC POTENTIALS}

In this appendix, we derive the harmonic oscillator expression for the power spectrum intensity of Eq. (15), i.e., for 
a reference state given by the combination of coherent states. To calculate the coherent states overlap in Eq. (14), one needs to insert the space indentity twice, use Eq. (7) and perform the space integration. For an $\mathrm{i}$-th versus $\mathrm{j}$-th coherent state overlap we obtain the following expression (for $\hbar=1$ ),

$$
\begin{aligned}
& \left\langle p_{j}, q_{j} \mid p_{t_{2}}, q_{t_{2}}\right\rangle\left\langle p_{t_{1}}, q_{t_{1}} \mid p_{i}, q_{i}\right\rangle \\
& =\exp \left[-\frac{\gamma}{4}\left(q_{i}-q_{t_{1}}\right)^{2}-\frac{\gamma}{4}\left(q_{j}-q_{t_{2}}\right)^{2}\right. \\
& \quad-\frac{1}{4 \gamma}\left(p_{i}-p_{t_{1}}\right)^{2}-\frac{1}{4 \gamma}\left(p_{j}-p_{t_{2}}\right)^{2}-\frac{i}{2}\left(q_{i}-q_{t_{1}}\right)\left(p_{i}-p_{t_{1}}\right) \\
& \quad+\frac{i}{2}\left(q_{j}-q_{t_{2}}\right)\left(p_{j}-p_{t_{2}}\right) .
\end{aligned}
$$

In the harmonic oscillator case, the following equation of motion in mass-scaled form can be employed:

$$
\begin{gathered}
\mathbf{q}(t)=\mathbf{q}(0) \cos (\omega t)+\frac{\mathbf{p}(0)}{\omega} \sin (\omega t), \\
\mathbf{p}(t)=\mathbf{p}(0) \cos (\omega t)-\omega \mathbf{q}(0) \sin (\omega t),
\end{gathered}
$$

and the difference of the corresponding actions to insert into Eq. (14) is

$$
\begin{aligned}
S\left(t_{2}\right)-S\left(t_{1}\right)= & \mathbf{p}(0) \mathbf{q}(0)\left(\cos ^{2}\left(\omega t_{2}\right)-\cos ^{2}\left(\omega t_{1}\right)\right) \\
& +\left(\frac{\mathbf{p}^{2}(0)}{2 \omega}-\frac{\omega \mathbf{q}^{2}(0)}{2}\right)\left(\cos \left(\omega t_{2}\right) \sin \left(\omega t_{2}\right)\right. \\
& \left.-\cos \left(\omega t_{1}\right) \sin \left(\omega t_{1}\right)\right) .
\end{aligned}
$$

When setting $\gamma=\omega$, substituting the harmonic equation of motion Eqs. (A2) and (A3) into (A1), and considering that the prefactor of Eq. (6) has the following expression for the harmonic oscillator:

$$
C_{t_{2}}\left(\mathbf{p}\left(t_{1}\right), \mathbf{q}\left(t_{1}\right)\right)=\mathrm{e}^{i \omega\left(t_{2}-t_{1}\right) / 2},
$$

then Eq. (14) becomes

$$
\begin{aligned}
I(E)= & \sum_{i, j} e^{-\omega\left(q_{j}^{2}+q_{i}^{2}\right) / 4-\left(p_{i}^{2}+p_{j}^{2}\right) / 4 \omega-i\left(p_{i} q_{i}-p_{j} q_{j}\right) / 2} \\
& \times \frac{\operatorname{Re}}{\pi T} \int_{0}^{T} d t_{1} \int_{t_{1}}^{+\infty} d t_{2} e^{i E\left(t_{2}-t_{1}\right)-i \omega\left(t_{2}-t_{1}\right) / 2} \\
& \times \frac{1}{2 \pi} \iint d p_{0} d q_{0} e^{-\omega q_{0}^{2} / 2-p_{0}^{2} / 2 \omega} \exp \left[\frac { q _ { 0 } } { 2 } \left(\omega q_{i} \cos \left(\omega t_{1}\right)\right.\right. \\
& \left.+\omega q_{j} \cos \left(\omega t_{2}\right)-p_{i} \sin \left(\omega t_{1}\right)-p_{j} \sin \left(\omega t_{2}\right)\right) \\
& +\frac{p_{0}}{2}\left(q_{i} \sin \left(\omega t_{1}\right)+q_{j} \sin \left(\omega t_{2}\right)+\frac{p_{i}}{\omega} \cos \left(\omega t_{1}\right)\right. \\
& \left.\left.+\frac{p_{j}}{\omega} \cos \left(\omega t_{2}\right)\right)\right] \exp \left[\frac { i } { 2 } q _ { 0 } \left(\omega q_{i} \sin \left(\omega t_{1}\right)\right.\right. \\
& \left.+p_{i} \cos \left(\omega t_{1}\right)-\omega q_{j} \sin \left(\omega t_{2}\right)-p_{j} \cos \left(\omega t_{2}\right)\right) \\
& +\frac{i}{2} p_{0}\left(\frac{p_{i}}{\omega} \sin \left(\omega t_{1}\right)-q_{i} \cos \left(\omega t_{1}\right)-\frac{p_{j}}{\omega} \sin \left(\omega t_{2}\right)\right. \\
& \left.\left.+q_{j} \cos \left(\omega t_{2}\right)\right)\right]
\end{aligned}
$$

By performing Guassian integrals in $p_{0}$ and $q_{0}$, Eq. (A6) is left with the following double-time integration:

$$
\begin{aligned}
I(E)= & \sum_{i, j} e^{-\omega\left(q_{j}^{2}+q_{i}^{2}\right) / 4-\left(p_{i}^{2}+p_{j}^{2}\right) / 4 \omega-i\left(p_{i} q_{i}-p_{j} q_{j}\right) / 2} \\
& \times \frac{\operatorname{Re}}{\pi T} \int_{0}^{T} d t_{1} \int_{t_{1}}^{+\infty} d t_{2} e^{i E\left(t_{2}-t_{1}\right)-i \omega\left(t_{2}-t_{1}\right) / 2} \\
& \times \exp \left[\frac { 1 } { 2 \omega } \left(\omega^{2} q_{i} q_{j} \cos \left(\omega\left(t_{2}-t_{1}\right)\right)+\omega q_{i} p_{j} \sin \left(\omega\left(t_{2}-t_{1}\right)\right)\right.\right. \\
& \left.-\omega q_{j} p_{i} \sin \left(\omega\left(t_{2}-t_{1}\right)\right)+p_{i} p_{j} \cos \left(\omega\left(t_{2}-t_{1}\right)\right)\right) \\
& +\frac{i}{2 \omega}\left(-\omega^{2} q_{i} q_{j} \sin \left(\omega\left(t_{2}-t_{1}\right)\right)-p_{i} p_{j} \sin \left(\omega\left(t_{2}-t_{1}\right)\right)\right. \\
& \left.\left.-\omega q_{i} p_{j} \cos \left(\omega\left(t_{2}-t_{1}\right)\right)+\omega q_{j} p_{i} \cos \left(\omega\left(t_{2}-t_{1}\right)\right)\right)\right] .
\end{aligned}
$$

After changing variables, $\tau=t_{2}-t_{1}$, and collecting the sine and cosine terms, the time integration in Eq. (A7) becomes

$$
\begin{aligned}
I(E)= & \sum_{i, j,} e^{-\omega\left(q_{j}^{2}+q_{i}^{2}\right) / 4-\left(p_{i}^{2}+p_{j}^{2}\right) / 4 \omega-i\left(p_{i} q_{i}-p_{j} q_{j}\right) / 2} \\
& \times \frac{\operatorname{Re}}{\pi} \int_{0}^{+\infty} d \tau e^{i \tau(E-\omega / 2)} \\
& \times \exp \left[\left(\frac{\omega}{2} q_{i} q_{j}+\frac{p_{i} p_{j}}{2 \omega}-\frac{q_{i} q_{j}}{2}+\frac{q_{j} p_{i}}{2}\right) e^{-i \omega \tau}\right] .
\end{aligned}
$$

By expanding the squared brackets exponential part and performing the time integration, one obtains Eq. (15) in the main text.

${ }^{1}$ H. Wang and M. Thoss, J. Chem. Phys. 119, 1289 (2003).

${ }^{2}$ H. Wang, D. E. Skinner, and M. Thoss, J. Chem. Phys. 125, 174502 (2006).

${ }^{3}$ J. M. Bowman, B. J. Braams, S. Carter, C. Chen, G. Czako, B. Fu, X. Huang, E. Kamarchik, A. R. Sharma, B. C. Shepler, Y. Wang, and Z. Xie, J. Phys. Chem. Lett. 1, 1866 (2010).

${ }^{4}$ J. M. Herbert and M. Head-Gordon, Phys. Chem. Chem. Phys. 7, 3269 (2005).

${ }^{5}$ A. Odell, A. Delin, B. Johansson, N. Bock, M. Challacombe, and A. M. N. Niklasson, J. Chem Phys. 131, 244106 (2009).

${ }^{6}$ A. M. N. Niklasson, C. J. Tymczak, and M. Challacombe, Phys. Rev. Lett. 97, 123001 (2006); J. Chem. Phys. 126, 144103 (2007).

${ }^{7}$ B. Q. Li, C. Mollica, and J. Vanicek, J. Chem. Phys. 131, 041101 (2009).

${ }^{8}$ T. Zimmermann, J. Ruppen, B. Q. Li, and J. Vanicek, Int. J. Quantum Chem. 110, 2426 (2010).

${ }^{9}$ R. Car and M. Parrinello, Phys. Rev. Lett. 55, 2471 (1985).

${ }^{10}$ T. D. Kuhne, M. Krack, F. R. Mohamed, and M. Parrinello, Phys. Rev. Lett. 98, 066401 (2007).

${ }^{11}$ H. B. Schlegel, J. M. Millam, S. S. Iyengar, G. A. Voth, A. D. Daniels, G. E. Scuseria, and M. J. Frisch, J. Chem. Phys. 114, 9758 (2001).

${ }^{12}$ J. M. Herbert and M. Head-Gordon, J. Chem. Phys. 121, 11542 (2004).

${ }^{13}$ Y. Liu, D. Yarne, and M. E. Tuckerman, Phys. Rev. B 68, 125110 (2003).

${ }^{14}$ I. Summer and S. S. Iyengar, J. Phys. Chem A 111, 10313 (2007).

${ }^{15}$ O. Knospe and P. Jungwirth, Chem. Phys. Lett. 317, 529 (2000).

${ }^{16}$ M. Ben-Nun and T. J. Martinez, Adv. Chem. Phys. 121, 439 (2002); B. G. Levine, J. D. Coe, A. M. Virshup, and Todd J. Martinez, Chem. Phys. 347, 3 (2008); J. D. Coe, B. G. Levine, and T. J. Martinez, J. Phys. Chem. 111, 11302 (2007)

${ }^{17}$ H.-D. Meyer, F. Gatti, and G. A. Worth, Multidimensional Quantum Dynamics: $M C T D H$ Theory and Applications (Wiley-VCH, Weinheim, 2009). 
${ }^{18}$ I. Kassal, S. P. Jordan, P. J. Love, and A. Aspuru-Guzik, Proc. Nat. Acad. Sci. U.S.A. 105, 18681 (2008).

${ }^{19}$ D. W. Lu, N. Y. Xu, R. X. Xu, H. W. Chen, J. B. Gong, J. X. H. Peng, and J. F. Du, Phys. Rev. Lett. 107, 020501 (2011).

${ }^{20}$ M. Ceotto, S. Atahan, S. Shim, G. F. Tantardini, and A. Aspuru-Guzik, Phys. Chem. Chem. Phys. 11, 3861 (2009).

${ }^{21}$ M. Ceotto, S. Atahan, G. F. Tantardini, and A. Aspuru-Guzik, J. Chem. Phys. 130, 234113 (2009).

${ }^{22}$ J. Tatchen and E. Pollak, J. Chem. Phys. 130, 041103 (2009).

${ }^{23}$ S. Y. Y. Wong, D. M. Benoit, M. Lewerenz, A. Brown, and P.-N. Roy, J. Chem. Phys. 134, 094110 (2011).

${ }^{24}$ W. H. Miller, J. Chem. Phys. 53, 3578 (1970); ibid. 53, 1949 (1970); W. H. Miller, J. Phys. Chem. A 105, 2942 (2001); W. H. Miller, Adv. Chem. Phys. 25, 69 (1974).

${ }^{25}$ E. J. Heller, J. Chem. Phys. 62, 1544 (1975); ibid. 75, 2923 (1981).

${ }^{26}$ M. F. Herman, Annu. Rev. Phys. Chem. 45, 83 (1994).

${ }^{27}$ K. G. Kay, Annu. Rev. Phys. Chem. 56, 255 (2005).

${ }^{28}$ D. J. Tannor and S. Garashchuk, Annu. Rev. Phys. Chem. 51, 553 (2000).

${ }^{29}$ M. Thoss and H. Wang, Annu. Rev. Phys. Chem. 55, 299 (2004).

${ }^{30}$ D. J. Tannor, Introduction to Quantum Mechanics: A Time-dependent Perspective (University Science Books, Sausalito, California, 2007).

${ }^{31}$ E. J. Heller, Acc. Chem. Res. 14, 368 (1981).

${ }^{32}$ A. L. Kaledin and W. H. Miller, J. Chem. Phys. 118, 7174 (2003); A. L. Kaledin and W. H. Miller, J. Chem. Phys. 119, 3078 (2003).

${ }^{33}$ D. V. Shalashilin and M. S. Child, J. Chem. Phys. 115, 5367 (2001).

${ }^{34}$ Y. Elran and K. G. Kay, J. Chem. Phys. 110, 3653 (1999); ibid. 110, 8912 (1999).

${ }^{35}$ H. Wang, X. Sun, and W. H. Miller, J. Chem. Phys. 108, 9726 (1988); X. Sun and W. H. Miller, ibid. 110, 6635 (1999); M. Thoss, H. Wang, and W. H. Miller, ibid. 114, 9220 (2001); T. Yamamoto, H. Wang, and W. H. Miller, ibid. 116, 7335 (2002); T. Yamamoto and W. H. Miller, ibid. 118, 2135 (2003).

${ }^{36} \mathrm{M}$. Topaler and N. Makri, J. Chem. Phys. 101, 7500 (1994); K. Thompsoon and N. Makri, J. Chem. Phys. 110, 1343 (1999); N. Makri, Annu. Rev. Phys. Chem. 50, 167 (1999); N. J. Wright and N. Makri, J. Chem. Phys. 119, 1634 (2003).

${ }^{37}$ J. Ankerhold, M. Saltzer, and E. Pollak, J. Chem. Phys. 116, 5925 (2002); S. Zhang and E. Pollak, Phys. Rev. Lett. 91, 190201 (2003); S. S. Zhang and E. Pollak, J. Chem. Phys. 121, 3384 (2004).

${ }^{38}$ A. R. Walton and D. E. Manolopoulos, Mol. Phys. 87, 961 (1996); A. R. Walton and D. E. Manolopoulos, Chem. Phys. Lett. 244, 448 (1995); M. L. Brewer, J. S. Hulme, and D. E. Manolopoulos, J. Chem. Phys. 106, 4832 (1997).
${ }^{39}$ S. Bonella, D. Montemayor, and D. F. Coker, Proc. Natl. Am. Soc. 102, 6715 (2005); S. Bonella and D. F. Coker, J. Chem. Phys. 118, 4370 (2003).

${ }^{40}$ Y. Wu , M. Herman, and V. S. Batista, J. Chem. Phys. 122, 114114 (2005); Y. Wu and V. S. Batista, J. Chem. Phys. 118, 6720 (2003).

${ }^{41}$ F. Grossmann, Comments At. Mol. Phys. 34, 243 (1999); C.-M. Goletz and F. Grossmann, J. Chem. Phys. 130, 244107 (2009).

${ }^{42}$ H. Ushiyama and K. Takatsuka, J. Chem. Phys. 122, 224122 (2005).

${ }^{43}$ G. Hochman and K. G. Kay, J. Chem. Phys. 130, 061104 (2009).

${ }^{44}$ B. B. Issack and P. N. Roy, J. Chem. Phys. 127, 054105 (2007).

${ }^{45}$ J. Liu and W. H. Miller, J. Chem. Phys. 125, 224104 (2006); ibid. 127, 114506 (2007); ibid. 128, 144511 (2008).

${ }^{46}$ B. R. McQuarrie, D. G. Abrashkevich, and P. Brumer, J. Chem. Phys. 119, 3606 (2003).

${ }^{47}$ M. F. Herman and E. Kluk, Chem. Phys. 91, 27 (1984); K. G. Kay, J. Chem. Phys. 100, 4377 (1994); K. G. Kay, J. Chem. Phys. 100, 4432 (1994).

${ }^{48}$ H. Wang, D. E. Manolopoulos, and W. H. Miller, J. Chem. Phys. 115, 6317 (2001).

${ }^{49}$ D. Huber and E. J. Heller, J. Chem. Phys. 89, 4752 (1988); D. Huber, E. J. Heller, and R. G. Littlejohn, ibid. 89, 2003 (1998); D. Huber, S. Ling, D. G. Imre, and E. J. Heller, ibid. 90, 7317 (1989); J. C. Burant and V. S. Batista, ibid. 116, 2748 (2002); D. V. Shalashilin, M. S. Child, and D. C. Clary, ibid. 120, 5608 (2004).

${ }^{50}$ M. Ceotto, D. dell'Angelo, and G. F. Tantardini, J. Chem. Phys. 133, 054701 (2010).

${ }^{51}$ M. Ceotto, S. Valleau, G. F. Tantardini, and A. Aspuru-Guzik, J. Chem Phys. 134, 234103 (2011).

${ }^{52}$ G. C. Carney, L. L. Sprandel, and C. W. Kern, Adv. Chem. Phys. 37, 305 (1978).

${ }^{53}$ J. M. Bowman, J. Chem. Phys. 68, 608 (1978).

${ }^{54}$ J. M. Bowman, K. M. Christoffel, and F. L. Tobin, J. Phys. Chem. 83, 905 (1979).

${ }^{55}$ K. M. Christoffel and J. M. Bowman, Chem. Phys. Lett. 85, 220 (1982).

${ }^{56}$ S. Carter, J. M. Bowman, and N. C. Handy, Theor. Chem. Acc. 100, 191 (1988).

${ }^{57}$ Y. Shao, L. Fusti Molnar, Y. Jung et al., Phys. Chem. Chem. Phys. 8, 3172 (2006).

${ }^{58}$ R. Gelabert, X. Giménez, M. Thoss, H. Wang, and W. H. Miller, J. Phys. Chem A 104, 10321 (2000).

${ }^{59}$ B. R. Johnson, Chem. Phys. 2, 381 (1973).

${ }^{60}$ P.-N. Roy and A. Brown, private communication (2011)

${ }^{61}$ B. Harland and P.-N. Roy, J. Chem. Phys. 118, 4791 (2003).

${ }^{62}$ B. B. Issack and P.-N. Roy, J. Chem. Phys. 123, 084103 (2005).

${ }^{63}$ B. B. Issack and P.-N. Roy, J. Chem. Phys. 127, 144306 (2007).

${ }^{64}$ B. B. Issack and P.-N. Roy, J. Chem. Phys. 126, 024111 (2007). 